\title{
Hyperaldosteronism and altered expression of an SGK1-dependent sodium transporter in ZDF rats leads to salt dependence of blood pressure
}

\author{
Markus Resch ${ }^{1}$, Tobias Bergler ${ }^{1}$, Sabine Fredersdorf ${ }^{1}$, Daniel P Griese ${ }^{1}$, Joachim Weil ${ }^{2}$, Peter Kreuzer ${ }^{1}$, \\ Sabine Brunner ${ }^{1}$, Günter AJ Riegger ${ }^{1}$, Andreas Luchner ${ }^{1}$ and Dierk H Endemann ${ }^{1}$
}

This study was designed to test whether altered aldosterone-related sodium handling leads to salt-sensitive blood pressure in diabetes and thus may exaggerate end-organ damage. Zucker diabetic fatty (ZDF) rats, a model of type 2 diabetes, and Zucker lean (ZL) rats, as euglycemic controls, were divided into groups receiving normal $(0.28 \%)(Z D F+N, Z L+N)$ and high-salt $(5.5 \%)$ diets (ZDF+S, ZL+S) for 10 weeks. Renal mRNA expression of serum- and glucocorticoid-inducible kinase 1 (SGK1) and sodium transporters (for example, the epithelial sodium channel- $\alpha, \mathrm{ENaC} \alpha$ ) were measured by quantitative reverse transcriptase-PCR. Vascular hypertrophy (media-to-lumen ratio, $M / L$ ) in mesenteric resistance arteries was assessed using a pressurized myograph. Systolic blood pressure (SBP) was significantly higher in ZDF+S vs. ZDF+N (146 \pm 2 vs. $133 \pm 3 \mathrm{~mm} \mathrm{Hg} ; P<0.05)$, whereas there was no difference between $Z L+S$ and $Z L+N(151 \pm 3$ vs. $147 \pm 3 \mathrm{~mm} \mathrm{Hg})$. Plasma sodium concentration was higher in $\mathrm{ZDF}+\mathrm{S}$ vs. $\mathrm{ZDF}+\mathrm{N}$, whereas there was no difference between $\mathrm{ZL}+\mathrm{S}$ and $\mathrm{ZL}+\mathrm{N}$. Plasma aldosterone concentration (PAC) was higher in ZDF+N as compared with ZL+N (191 \pm 23 vs. $\left.95 \pm 35 \mathrm{pg} \mathrm{ml}^{-1} ; P<0.05\right)$. PAC decreased to zero in ZL+S, which was not the case in ZDF+S $\left(0 \pm 0\right.$ vs. $\left.37 \pm 2 \mathrm{pg} \mathrm{ml}^{-1}\right)$. Salt loading decreased the mRNA expression of SGK1 in euglycemic controls ( $\mathrm{ZL}+\mathrm{S} 0.58 \pm 0.2$ vs. $\mathrm{ZL}+\mathrm{N} 1.05 \pm 0.05 ; P=0.05)$, whereas it significantly increased $\mathrm{SGK} 1$ expression in diabetic rats (ZDF+S $1.75 \pm 0.15$ vs. $Z D F+N \quad 0.92 \pm 0.07 ; P<0.01)$. ENaC $\alpha$ mRNA expression paralleled these changes. The M/L of mesenteric resistance arteries was not different between $Z D F+N$ and $Z L+N$. High salt significantly increased the $M / L$ in $Z D F+S$ vs. ZDF+N, but not in ZL+S vs. ZL+N. Systolic blood pressure in this model of type 2 diabetes mellitus is salt sensitive, leading to marked vascular remodeling. The underlying pathophysiological mechanism may be inappropriately high levels of aldosterone and up-regulation of SGK1-dependent renal sodium transport by $\mathrm{ENaC}_{\alpha}$, leading to net increased sodium retention.

Hypertension Research (2010) 33, 1082-1088; doi:10.1038/hr.2010.132; published online 22 July 2010

Keywords: EnaC; salt-sensitive blood pressure; SGK1

\section{INTRODUCTION}

Diabetes mellitus and arterial hypertension are important causes of vascular damage. The combination of the two places the patient at particularly high cardiovascular risk. ${ }^{1}$ Both diabetes mellitus and hypertension are part of the metabolic syndrome. However, it is not fully understood how they influence each other. In this context, some studies suggest that blood pressure (BP) in diabetes mellitus is salt sensitive. ${ }^{2-4}$ Recently, a study in 1906 Chinese participants suggested that the metabolic syndrome enhances the BP response to sodium intake. ${ }^{5}$ However, the underlying pathological mechanisms remain to be elucidated. Aldosterone is known to have a major function in the regulation of salt balance, and we have previously shown that Zucker diabetic fatty (ZDF) rats, as a model for type 2 diabetes mellitus, display hyporeninemic hyperaldosteronism. ${ }^{6}$ Induction of the serumand glucocorticoid-induced kinase 1 (SGK1), an aldosterone target gene, is a primary event in the antinatriuretic effect of aldosterone., The activation of SGK1 has been reported to be carried out mainly by phosphoinositide-dependent kinase, a downstream regulator of phosphoinositide 3-kinase., ${ }^{9,10}$ One of the known actions of SGK1 is to activate the $\alpha$-subunit of the epithelial $\mathrm{Na}^{+}$channel (ENaC), a key regulator of $\mathrm{Na}^{+}$transport in the kidney and other epithelia, resulting in enhanced sodium reabsorption in the cortical collecting tubules. ${ }^{8}$ Furthermore, SGK1 regulates the plasma membrane abundance of $\mathrm{ENaC}$ by interaction with and phosphorylation of Nedd4-2, a downregulator of $\mathrm{ENaC}$, leading to reduced interaction between $\mathrm{ENaC}$ and Nedd4-2 and thus elevated ENaC cell surface expression. ${ }^{11,12}$ Thus, naturally occurring Nedd4-2 isoforms differentially associate with $\mathrm{ENaC}$, regulating its activity. ${ }^{13}$ Besides $\mathrm{ENaC}$, the thiazide-sensitive $\mathrm{NaCl}$ cotransporter (NCC) is activated by aldosterone, also through a SGK1-dependent pathway. ${ }^{14}$

${ }^{1}$ Department of Internal Medicine II, University Medical Center Regensburg, Regensburg, Germany and ${ }^{2}$ Department of Internal Medicine II, School of Medicine, University Hospital, Lübeck, Germany

Correspondence: Dr M Resch, Department of Internal Medicine II, University Medical Center Regensburg, Franz Josef Strauss Allee 11, Regensburg D-93042, Germany. E-mail: Markus.Resch@klinik.uni-regensburg.de

Received 7 December 2009; revised 29 May 2010; accepted 30 May 2010; published online 22 July 2010 
In addition to aldosterone, hyperinsulinemia has been shown to phosphorylate SGK1 by a phosphoinositide 3-kinase pathway and may therefore lead to sodium reabsorption. ${ }^{15-18}$

Given the elevated aldosterone and insulin levels in ZDF rats, we hypothesized that renal SGK1 expression would be increased in experimental type 2 diabetes mellitus, leading to salt-sensitive hypertension and early remodeling of resistance arteries. To address this hypothesis, we studied ZDF rats on normal- and high-salt diets. ZDF rats have a mutation in the leptin receptor-encoding gene that impairs the ability of leptin to suppress food intake, making them a useful model of type 2 diabetes and the metabolic syndrome. Their Zucker lean (ZL) littermates on normal- and high-salt diets served as controls. All animals were characterized with respect to systolic blood pressure (SBP), renal sodium excretion, aldosterone, renal SGK1, the expression of $\mathrm{ENaC}$ and other sodium cotransporters (for example, NCC) and vascular remodeling. Most complications of diabetes mellitus and arterial hypertension are related to vascular damage. Moreover, remodeling of small resistance arteries has been suggested to be the earliest form of end-organ damage in mild essential hypertension. ${ }^{19}$ For small resistance arteries, the media-to-lumen ratio, a measure of vascular remodeling, has been shown to be a strong predictor of cardiovascular end points. ${ }^{20}$ Thus, vascular remodeling was studied in small resistance arteries in this experiment using a small vessel myograph.

\section{METHODS}

\section{Animal experiments}

The study was approved by the local committee on animal research and adhered to the 'Guide for the Care and Use of Laboratory Animals' published by the US National Institutes of Health. Eighteen male ZDF rats ( $f a / f a$ ) (Charles River Laboratories, Wilmington, MA, USA) with a genetically induced form of type 2 diabetes, including severe hyperglycemia, and 18 normoglycemic ZL controls (Charles River Laboratories) were studied from age 14 weeks to 25 weeks. Rats were fed Purina 5008 rat chow (Charles River Laboratories) containing $23 \%$ protein, $6.5 \%$ fat, $58.5 \%$ carbohydrates, $4 \%$ fiber and $8 \%$ ash. They received tap water ad libitum. Rats were individually housed on a 12$\mathrm{h}$ dark/12-h light cycle. At 15 weeks, rats were divided into four groups: ZDF rats receiving normal-salt diet $(0.28 \% \mathrm{NaCl})(n=9)(\mathrm{ZDF}+\mathrm{N}), \mathrm{ZDF}$ rats receiving high-salt diet $(5.5 \% \mathrm{NaCl}$ in chow) $(n=9)(\mathrm{ZDF}+\mathrm{S}), \mathrm{ZL}$ rats receiving normal-salt diet $(0.28 \% \mathrm{NaCl})(n=9)(\mathrm{ZL}+\mathrm{N})$ and $\mathrm{ZL}$ rats receiving high-salt $\operatorname{diet}(5.5 \% \mathrm{NaCl}$ in chow) $(n=9)(\mathrm{ZL}+\mathrm{S})$. Rats were housed in metabolic cages at weeks 13, 15, 19 and 24 to assess 24 -h excretion of sodium. Blood glucose was measured weekly from an incision in the tail vein (Accu-Chek Sensor, Roche, Mannheim, Germany). SBP and heart rate were measured weekly by the tail cuff method using an automated cuff inflator-pulse system (W+W electronic AG, BPrecorder No. 8005, Basel, Switzerland). At the end of the experiment, animals were killed and the mesenteric vasculature dissected. One segment was used for preparation of small arteries. Kidneys were removed, weighed and immediately snap-frozen in liquid nitrogen and stored at $-80{ }^{\circ} \mathrm{C}$ for further mRNA and protein analysis.

\section{Preparation and study of small arteries}

A third-order branch of the mesenteric artery was carefully dissected and mounted on a pressurized myograph (111P, Danish Myo Technology, Aarhus, Denmark). Perfusion was performed using oxygenated $37^{\circ} \mathrm{C}$ Krebs solution $\left(95 \% \mathrm{O}_{2}, 5 \% \mathrm{CO}_{2}\right)$ pH 7.4 containing $\left(\mathrm{mmoll}^{-1}\right)$ : $\mathrm{NaCl}(118), \mathrm{KH}_{2} \mathrm{PO}_{4}$ (1.18), $\mathrm{KCl}$ (4.7), $\mathrm{MgSO}_{4}$ (1.18), $\mathrm{CaCl}_{2}$ (2.5), D-glucose (5.5), $\mathrm{NAHCO}_{3}$ (25) and EDTA (0.026). Vessels were equilibrated under a constant pressure of $45 \mathrm{~mm} \mathrm{Hg}$. The viability of the vessels was tested with a single dose of norepinephrine $\left(10^{-5} \mathrm{moll}^{-1}\right)$. After precontraction with $10^{-5} \mathrm{moll}^{-1}$ norepinephrine, endothelium-dependent relaxation was assessed with acetylcholine. Vascular morphology was assessed under resting conditions at three different points along the vessel. The growth index was calculated as $\left(\mathrm{CSA}_{h}-\mathrm{C}\right.$ $\left.\mathrm{SA}_{\mathrm{n}}\right) / \mathrm{CSA}_{\mathrm{n}}$, where $\mathrm{CSA}_{\mathrm{n}}$ and $\mathrm{CSA}_{\mathrm{h}}$ are the medial cross-sectional areas of normal-salt and high-salt vessels, respectively.

\section{Measurements in plasma and urine}

Plasma aldosterone concentration and plasma renin activity were measured using commercially available kits (DiaSorin, Dietzenbach, Germany) according to the manufacturer's instructions and as described elsewhere. ${ }^{21}$ The sampling was performed on ice. Plasma and urinary sodium were measured using a sodium-sensitive electrode (Advia 1800, Bayer Healthcare, Leverkusen, Germany). Plasma insulin levels were measured using a commercially available Insulin Elisa (Biocat GmbH, Heidelberg, Germany).

\section{RNA isolation, reverse transcription and real-time PCR in rat specimens}

After homogenization of frozen tissue sections in peqGOLD TriFast (Peqlab, Erlangen, Germany), total RNA was extracted according to the manufacturer's directions, with additional DNase digestion to remove all traces of genomic DNA.

Total RNA was reverse transcribed into complementary DNA according to standard protocols, as previously described. ${ }^{22}$ In brief, complementary DNA probes were synthesized in $20-\mu \mathrm{l}$ reaction volume containing $1 \mu \mathrm{g}$ total RNA, $0.5 \mu \mathrm{g}$ oligo $(\mathrm{dT})$ primer (Sigma-Aldrich, Munich, Germany), $40 \mathrm{U}$ RNasin (Promega, Mannheim, Germany), $0.5 \mathrm{~mm}$ dNTP (Amersham, Freiburg, Germany), $4 \mu 15 \times$ transcription buffer and $200 \mathrm{U}$ Moloney murine leukemia virus reverse transcriptase (Invitrogen, Karlsruhe, Germany) for $1 \mathrm{~h}$ at $37^{\circ} \mathrm{C}$. In parallel, no-reverse transcriptase and no-template controls were performed. Real-time quantitative reverse transcriptase-PCR was performed on an ABI PRISM 7900 detection system (Applied Biosystems, Darmstadt, Germany) using a QuantiTect SYBR Green PCR Kit (Qiagen, Hilden, Germany). Glyceraldehyde-3-phosphate dehydrogenase was used as reference gene. All water controls were negative for target and glyceraldehyde-3-phosphate dehydrogenase. Data were analyzed using SDS 2.2.2 software (Applied Biosystems). Primers are given in Table 1.

\section{Western blot}

SGK1 protein expression was examined in four representatives of each group by western blot analysis. After homogenization of frozen kidneys in peqGOLD TriFast (Peqlab Biotechnologies, Erlangen, Germany), total protein was extracted according to the manufacturer's directions. Protein concentrations were determined in duplicate with the method of Pierce. A bicinchoninic acid and copper sulfate mixture ( $200 \mu \mathrm{l} ; 50: 1)$ (Sigma-Aldrich) was combined with $10 \mu \mathrm{l}$ of the protein sample and incubated for $1 \mathrm{~h}$ at room temperature. Absorption was measured at $560 \mathrm{~nm}$. For western blot analysis, $25 \mu \mathrm{g}$ total protein was boiled with Laemmli buffer (Bio-Rad, Munich, Germany) at $95{ }^{\circ} \mathrm{C}$ for $5 \mathrm{~min}$, subjected to $12 \%$ SDS-polyacrylamide gel electrophoresis and then

\section{Table 1 Primer sequences for renal mRNA analysis}

\begin{tabular}{|c|c|}
\hline Gene & Sequence \\
\hline $\mathrm{ENaC} \alpha$ forw & 5'-CGTCACTGTCTGCACCCTA-3' \\
\hline $\mathrm{ENaC} \alpha$ rev & 5'-CCTGGCGAGTGTAGGAAGAG-3' \\
\hline $\mathrm{ENaC} \beta$ forw & 5'-GCAGAGGACATACCCCTTCA-3' \\
\hline $\mathrm{ENaC} \beta$ rev & 5'-CATGGTGCAGGGACTGTATG-3' \\
\hline $\mathrm{ENaC} \gamma$ forw & 5'-CTACCAGCAACACCCCAACT-3' \\
\hline $\mathrm{ENaC} \gamma$ rev & 5'-GCTACAGGATTGCTTGCACA-3' \\
\hline NCC forw & 5'-ACATTCTGGTGGTTGGCTTC-3' \\
\hline NCC rev & 5'-GCACACCCCGTAGTTGAAGT-3' \\
\hline NHE3 for & 5'-GGAGATCTTCCACAGGACCA-3' \\
\hline NHE3 rev & 5'-TGCTATTCCTCCGCTTCTGT-3' \\
\hline NKCC2 forw & 5'-TGTGAAGTTTGGATGGGTGA-3' \\
\hline NKCC2 rev & 5'-TGATGATGACTCCCAGACCA-3' \\
\hline SGK1 forw & 5'-ACGCAGCTGAAATAGCCAGT-3' \\
\hline SGK1 rev & 5'-TGTGCTCGATGTTCTCCTTG-3' \\
\hline GAPDH forw & 5'-GTCGTGGATCTGACGTGCC-3' \\
\hline GAPDH rev & 5'-GATGCCTGCTTCACCACCTT-3' \\
\hline
\end{tabular}


transferred to nitrocellulose membranes (Schleicher \& Schuell, Dassel, Germany) by semidry blotting, as described before. ${ }^{22}$ Membranes were blocked with Tris-buffered saline with $0.1 \%$ Tween-20 (TBST) containing 5\% non-fat dry milk for $90 \mathrm{~min}$ and incubated with the primary antibody (rabbit antiSGK1 $0.5 \mu \mathrm{g} \mathrm{ml}^{-1}$, Upstate-Millipore, Lake Placid, NY, USA) overnight at $4{ }^{\circ} \mathrm{C}$. A horseradish peroxidase-conjugated secondary antibody was used to detect immunoreactive bands using the enhanced chemiluminescence western blotting detection system (Amersham Bioscience, Buckinghamshire, UK). Membranes were exposed to Hyperfilm (Amersham Bioscience). Afterwards, nitrocellulose membranes were stripped, washed and blocked with TBST containing 5\% non-fat dry milk for $90 \mathrm{~min}$. Detection was performed with a goat anti- $\beta$-actin antibody (1:1000, Santa Cruz, Santa Cruz, CA, USA; overnight at $\left.4{ }^{\circ} \mathrm{C}\right)$ and a donkey anti-goat secondary antibody (1:2000, Santa Cruz; $1 \mathrm{~h}$ at room temperature) coupled to peroxidase and enhanced chemiluminescence.

\section{Statistical analysis}

Data are presented as mean \pm s.e.m. For blood glucose levels and SBP levels, mean values throughout active treatment were calculated for each animal. Groups were compared using one-way analysis of variance. Post hoc testing was performed using Fisher's least significant difference test. A $P$-value $<0.05$ was considered significant.

\section{RESULTS}

\section{Animal data}

Body weight was significantly lower in ZL rats compared with ZDF rats at the start of the study. Over time, ZL rats continued to grow, resulting in a nonsignificant difference by the end of the study (Table 2). Blood glucose was normal in all ZL rats, with no difference between the high- and normal-salt diets. ZDF rats displayed significantly elevated blood glucose levels throughout the study. Interestingly, blood glucose levels were significantly lower in $\mathrm{ZDF}+\mathrm{S}$ rats as compared with $\mathrm{ZDF}+\mathrm{N}$ rats on the normal diet (Figure 1). Confirming previous studies, SBP was lower in ZDF rats at the start of the treatment. Although salt loading significantly increased SBP in ZDF rats (Figure 2), it did not significantly change BP in normoglycemic $\mathrm{ZL}$ rats. There were no significant differences in heart rate throughout the study or in hematocrit at the end of the experiment (Table 2).

\section{Plasma and urinary analysis}

Serum sodium concentrations paralleled differences in mean SBP between the groups (Figure 3). Urinary volume was significantly higher in ZDF rats as compared with ZL rats. The high-salt diet significantly increased urinary volume in ZDF and ZL rats (Table 2).
The 24-h urinary sodium excretion was significantly increased under the high-salt diet and was higher in ZDF rats as compared with ZL rats. However, ZDF rats ate more, so estimated salt uptake by chow consumption was also higher in ZDF rats (Table 2). Plasma aldosterone concentration was significantly higher in ZDF rats as compared with controls (Table 2). In ZL rats, the high-salt diet reduced plasma aldosterone levels to zero. In ZDF rats, the high-salt diet also significantly reduced plasma aldosterone concentration, but not to zero. Plasma renin activity was lower in ZDF rats, confirming a lowrenin hyperaldosteronism in these animals. Salt loading significantly reduced plasma renin activity in ZL and ZDF rats (Table 2). Plasma insulin levels were higher in ZDF rats as compared with ZL rats, but at this age of the animals, the differences were not statistically different (Table 2).

\section{Structure of small resistance mesenteric arteries}

Thickness of the media was not different between ZL and ZDF rats. The high-salt diet did not significantly alter media thickness in normoglycemic ZL rats. However, the high-salt diet dramatically increased media thickness in ZDF rats (Figure 4c). There were no significant differences in lumen diameter between the groups (Figure 4d). However, lumen diameter tended to be lower in salt-

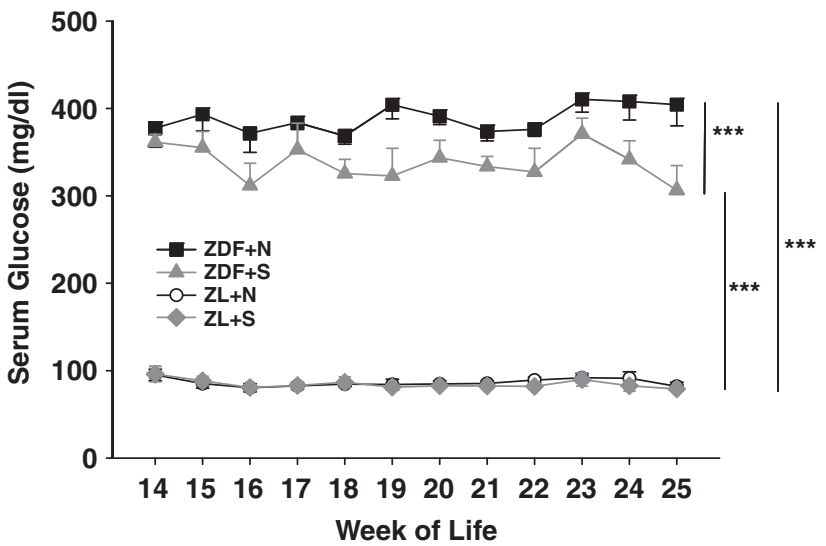

Figure 1 Serum glucose levels (mg per $100 \mathrm{ml}$ ) throughout the study period. Mean differences during treatment period: analysis of variance $P<0.001$, post hoc testing: ${ }^{* *} P<0.001$. Zucker lean $(\mathrm{ZL})+\mathrm{N}$ : $\mathrm{ZL}$ on normal diet; $\mathrm{ZL}+\mathrm{S}$ : ZL on high-salt diet; Zucker diabetic fatty (ZDF)+N: ZDF on normal diet; ZDF+S: ZDF on high-salt diet.

Table 2 Biometric, plasma and metabolic data

\begin{tabular}{|c|c|c|c|c|c|}
\hline & $Z L+N$ & $Z L+S$ & $Z D F+N$ & $Z D F+S$ & P-value \\
\hline Body weight at week $14(\mathrm{~g})$ & $325 \pm 5$ & $335 \pm 7$ & $340 \pm 13$ & $357 \pm 10$ & 0.11 \\
\hline Body weight at week 24 (g) & $381 \pm 7$ & $368 \pm 8$ & $362 \pm 13$ & $371 \pm 18$ & 0.75 \\
\hline Hematocrit & $44 \pm 2$ & $42 \pm 1$ & $43 \pm 2$ & $46 \pm 2$ & 0.57 \\
\hline PAC $\left(\mathrm{pg} \mathrm{ml}^{-1}\right)$ & $95 \pm 35$ & $0 \pm 0 * * *$ & $191 \pm 23^{*}$ & $37 \pm 12^{\dagger \dagger}$ & $<0.001$ \\
\hline PRA (ng ml ${ }^{-1} \mathrm{~h}^{-1}$ ) & $2.46 \pm 0.6$ & $0.2 \pm 0.2^{*}$ & $1.4 \pm 0.1$ & $0.0 \pm 0.0^{\dagger}$ & $<0.001$ \\
\hline Mean SBP over treatment period $(\mathrm{mm} \mathrm{Hg})$ & $146 \pm 2.6$ & $151 \pm 2.8$ & $133 \pm 3.0^{*}$ & $146 \pm 2.0^{\dagger}$ & $<0.001$ \\
\hline Mean heart rate over treatment period (b.p.m.) & $445 \pm 5$ & $443 \pm 3$ & $454 \pm 11$ & $455 \pm 15$ & 0.75 \\
\hline Insulin levels (ng ml ${ }^{-1}$ ) & $1.90 \pm 0.33$ & $1.45 \pm 0.19$ & $2.36 \pm 0.55$ & $1.86 \pm 0.39$ & 0.54 \\
\hline Urine volume ( $\mathrm{ml}$ per $24 \mathrm{~h}$ ) at week 24 & $12.3 \pm 1.5$ & $71.2 \pm 13.2^{* *}$ & $123.5 \pm 14.1^{* * *}$ & $215.7 \pm 20.8^{\dagger \dagger \dagger}$ & $<0.001$ \\
\hline Urinary sodium excretion ( $\mathrm{mmol}$ per $24 \mathrm{~h}$ ) at week 24 & $1.50 \pm 0.16$ & $16.87 \pm 2.88^{* * *}$ & $2.51 \pm 0.27$ & $25.60 \pm 2.60^{\dagger+\dagger}$ & $<0.001$ \\
\hline Urine volume ( $\mathrm{ml}$ per $24 \mathrm{~h}$ ) at week 24/body weight ( $\mathrm{g}$ ) at week 24 & $0.04 \pm 0.01$ & $0.16 \pm 0.03$ & $0.37 \pm 0.05^{* * *}$ & $0.61 \pm 0.07^{\dagger+\dagger}$ & $<0.001$ \\
\hline Estimated sodium uptake (mmol per $24 \mathrm{~h}$ ) at week 24 & $1.25 \pm 0.06$ & $19.05 \pm 1.13^{* * *}$ & $2.36 \pm 0.09$ & $28.57 \pm 1.23^{\dagger \dagger \dagger}$ & $<0.001$ \\
\hline
\end{tabular}

Abbreviations: PAC, plasma aldosterone concentration; PRA, plasma renin activity; ZDF, Zucker diabetic fatty; ZL, Zucker lean; ZL+N, ZL on normal diet; ZL+S, ZL on high-salt diet; ZDF+N, ZDF on normal diet; ZDF+S, ZDF on high-salt diet.

Post hoc testing: ${ }^{*} P<0.05$ vs. ZL+N, ${ }^{* *} P<0.01$ vs. ZL+N, ${ }^{* * *} P<0.001$ vs. $\mathrm{ZL}+\mathrm{N},{ }^{\dagger} P<0.05$ vs. ZDF+N, ${ }^{\dagger} P<0.01$ vs. ZDF+N, ${ }^{\dagger \dagger} P<0.001$ vs. ZDF+N. 

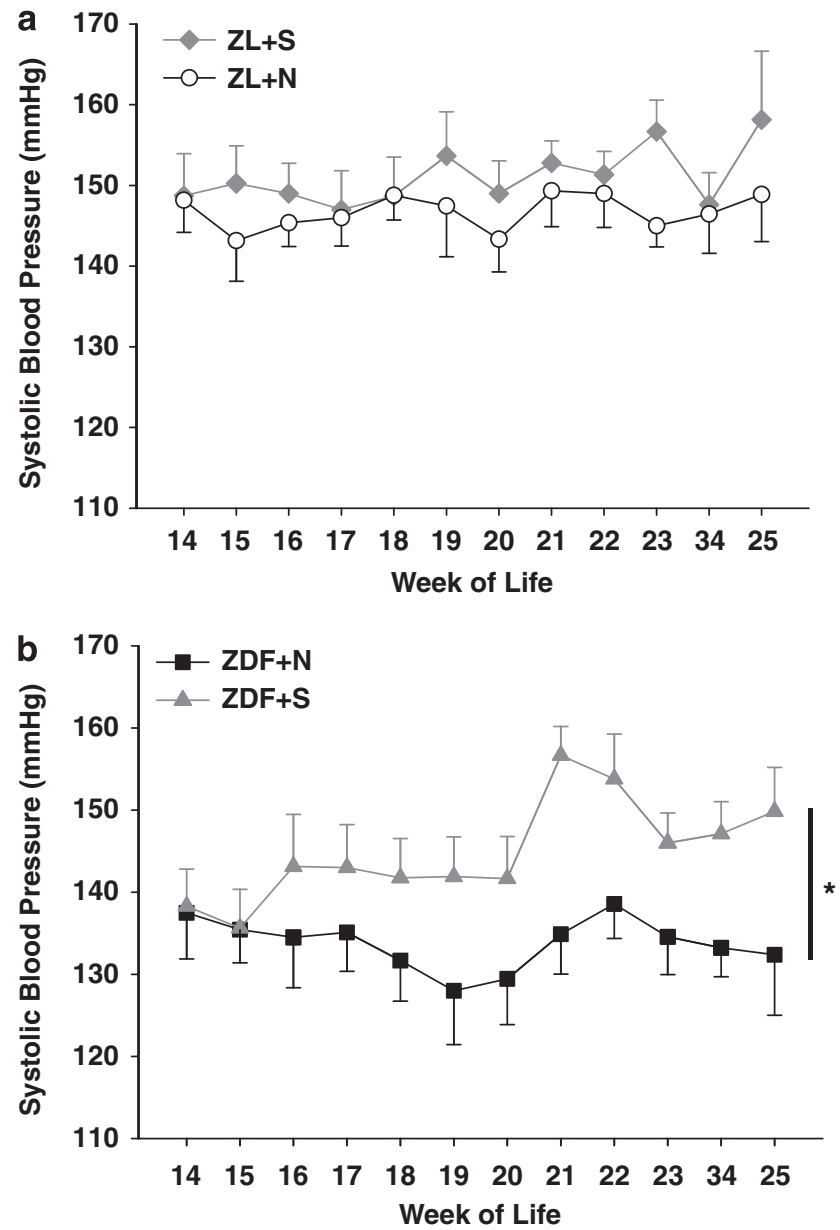

Figure 2 Systolic blood pressure values $(\mathrm{mm} \mathrm{Hg})$ throughout the study period in (a) Zucker lean (ZL) rats and (b) Zucker diabetic fatty (ZDF) rats. Mean differences during treatment period: analysis of variance $P<0.001$, post hoc testing: ${ }^{*} P<0.05 \mathrm{ZDF}+\mathrm{N}$ vs. $Z \mathrm{ZDF}+\mathrm{S}$. $\mathrm{ZL}+\mathrm{N}$ : $\mathrm{ZL}$ on normal diet; $\mathrm{ZL}+\mathrm{S}$ : ZL on high-salt diet; ZDF+N: ZDF on normal diet; ZDF+S: ZDF on high-salt diet.

treated ZDF rats, resulting in even more pronounced changes in the media-to-lumen ratio (Figure $4 \mathrm{a}$ ). The cross-sectional area tended to be higher in ZDF rats and increased with salt loading. However, these differences were not statistically significant (Figure $4 \mathrm{~b}$ ). The calculated growth index was identical for ZL and ZDF rats under salt treatment (33\% in both salt-loaded ZDF and salt-loaded ZL rats). This indicates marked inward vascular remodeling in salt-treated ZDF rats.

\section{SGK1 and SGK1-dependent epithelial sodium transporter mRNA expression}

In total kidney homogenates, mRNA expression levels of SGK1 were not significantly different between $\mathrm{ZL}+\mathrm{N}$ and $\mathrm{ZDF}+\mathrm{N}$ rats. As expected, mRNA levels were significantly decreased in normoglycemic $\mathrm{ZL}+\mathrm{S}$ rats. In contrast, mRNA levels were significantly increased in diabetic ZDF+S rats (Figure 5a). mRNA levels of the SGK1-dependent $\mathrm{ENaC} \alpha$ sodium channel paralleled the levels of SGK1 mRNA (Table 3). There was no evident regulation of the renal mRNA expression of $\mathrm{ENaC} \beta$ or $\mathrm{ENaC} \gamma$.

Confirming the mRNA data, we found a tremendous downregulation of SGK1 protein in ZL rats with high-salt treatment, which was not the case in ZDF rats (Figure $5 b$ ).

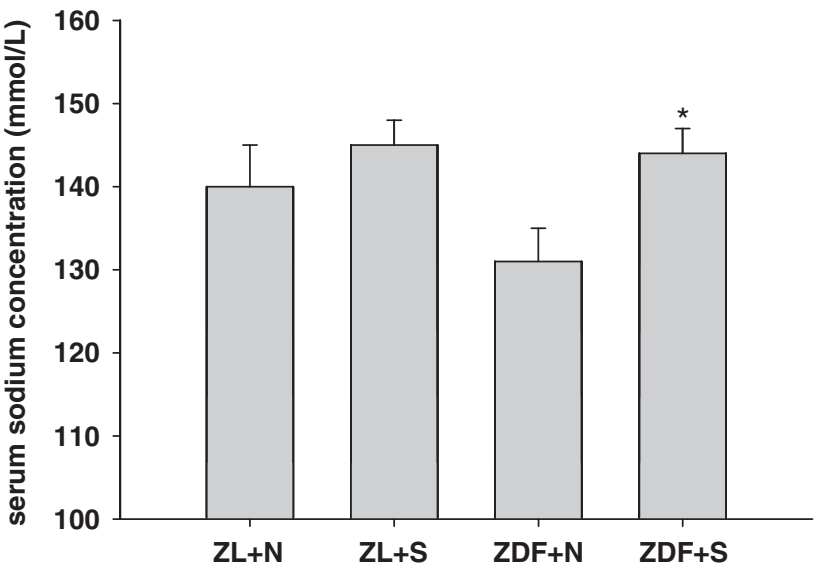

Figure 3 Serum sodium concentration $\left(\mathrm{mmol} \mathrm{I}^{-1}\right)$ at the end of the study. Analysis of variance $P=0.04$, post hoc testing: ${ }^{*} P<0.05$ Zucker diabetic fatty $(Z D F)+N$ vs. ZDF+S. Zucker lean (ZL)+N: ZL on normal diet; $Z L+S$ : ZL on high-salt diet; ZDF+N: ZDF on normal diet; ZDF+S: ZDF on high-salt diet.

Focusing on other renal sodium transporters (NCC, NKCC2, NHE3), we also found that NCC mRNA expression was significantly downregulated in $\mathrm{ZL}+\mathrm{S}$ rats. In $\mathrm{ZDF}+\mathrm{N}$ rats, NCC mRNA expression was lower than in $\mathrm{ZL}+\mathrm{N}$, but was not downregulated under high-salt treatment. A significant downregulation of NHE3 could only be found in $\mathrm{ZDF}+\mathrm{S}$ rats, whereas no significant difference in comparison with $\mathrm{ZL}+\mathrm{N}$ rats was found in the other groups $(\mathrm{ZL}+\mathrm{S}, \mathrm{ZDF}+\mathrm{N})$. We observed no effect on NKCC2 mRNA expression (Table 3).

\section{DISCUSSION}

In this study, we show that BP is salt sensitive in the ZDF rat model of type 2 diabetes mellitus. We also demonstrate that this salt sensitivity has a substantial impact on vascular remodeling in small resistance arteries, as an early form of end-organ damage. Specifically, the media-to-lumen quotient, which was increased by $60 \%$ under salt loading only in diabetic ZDF animals, has been shown to be a strong predictor of cardiovascular end points. ${ }^{20}$ Molecular analysis of the rat kidneys also showed dysregulation of $\mathrm{ENaC}$, perhaps leading to the observed salt sensitivity.

There is an ongoing debate about the causes of hypertension in the metabolic syndrome. In our study, the plasma sodium concentrations paralleled the mean SBP changes, suggesting that the BP changes are due to aldosterone-mediated sodium retention. First, we showed that aldosterone levels were elevated in ZDF rats, and second, we found that under high-salt treatment, aldosterone levels were significantly reduced, but not to zero, which was the case in salt-loaded normoglycemic controls. Thus, the remaining aldosterone level in the saltloaded ZDF rats may still have been pathologically high and thus may have caused sodium retention and promoted deleterious actions in combination with the high-salt status of these animals. Consistent with this hypothesis, in another study, aldosterone mainly induced end-organ damage in settings of high-salt loading. ${ }^{23}$ These findings may explain why diabetic ZDF rats under normal-salt conditions do not display vascular remodeling. It has been suggested that adipocytes produce aldosterone-releasing factors. ${ }^{24}$ It is intriguing to assume such a mechanism because plasma renin activity is reduced in ZDF rats. Moreover, it is suggested that aldosterone-releasing factor-induced aldosterone release is not suppressed by salt loading, which may explain why plasma aldosterone levels were not reduced to zero in ZDF rats on a high-salt diet. 

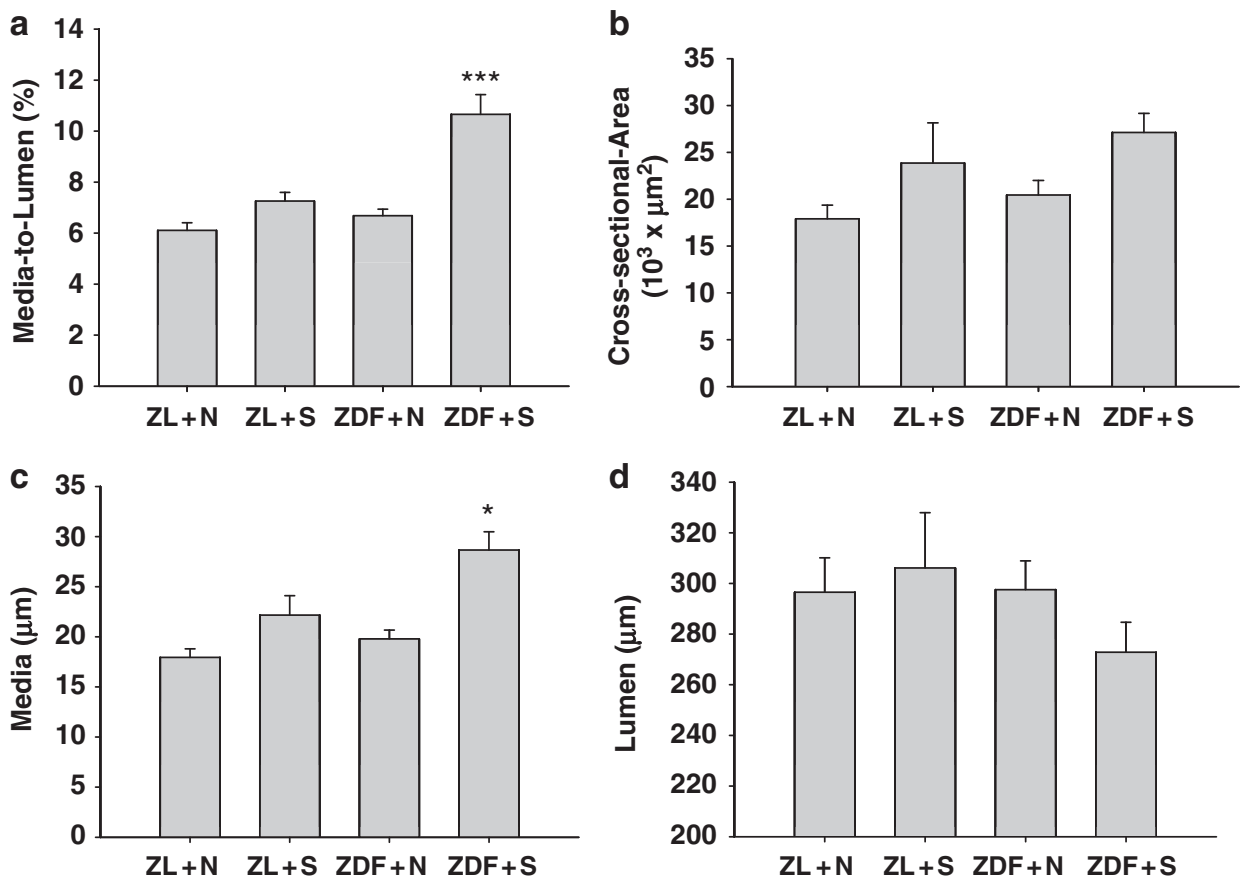

Figure 4 Structural characteristics of mesenteric resistance arteries. (a) Media-to-lumen ratio (\%), (b) cross-sectional area $\left(10^{3} \mu \mathrm{m}^{2}\right)$, (c) media $(\mu \mathrm{m})$ and (d) lumen $(\mu \mathrm{m})$. Analysis of variance media $P<0.001$, media-to-lumen ratio $P<0.001$, lumen $P=0.50$, cross-sectional area $P=0.1$, post hoc testing: ${ }^{*} P<0.05$ vs. all other groups, ${ }^{* *} P<0.001$ vs. all other groups. Zucker lean (ZL)+N: ZL on normal diet; ZL+S: ZL on high-salt diet; Zucker diabetic fatty $(Z D F)+N$ : ZDF on normal diet; ZDF+S: ZDF on high-salt diet.

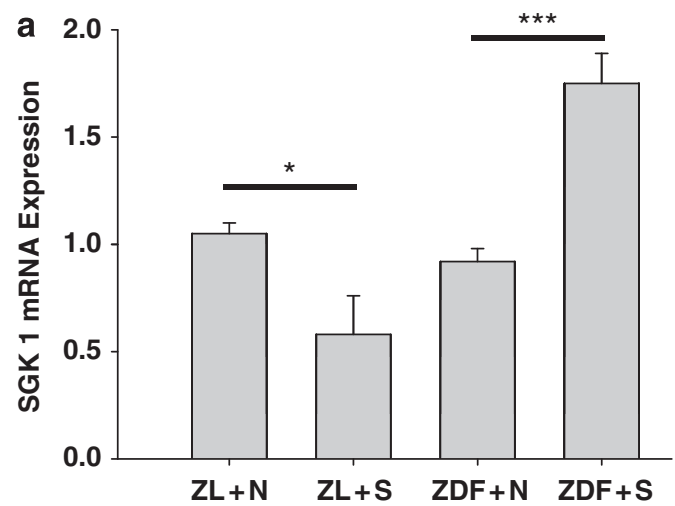

b
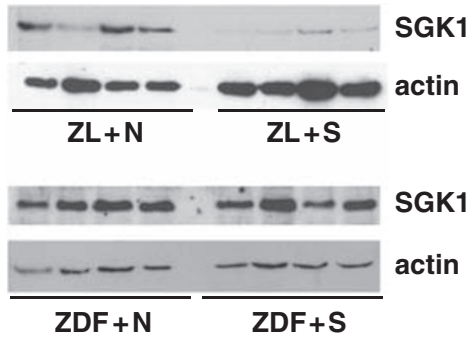

Figure 5 Renal mRNA and protein expression of serum- and glucocorticoid-inducible kinase 1 (SGK1). (a) Renal mRNA expression of (SGK1). Analysis of variance $P<0.001$, post hoc testing: ${ }^{*} P<0.05$ Zucker lean (ZL)+N vs. ZL+S, $* * * P<0.001$ Zucker diabetic fatty (ZDF) $+\mathrm{N}$ vs. ZDF+S. (b) Consequences of high-salt diet on ZL and ZDF SGK1 protein level relative to $\beta$-actin. The western blot analysis is a representative example of two independent experiments. $\mathrm{ZL}+\mathrm{N}$ : ZL on normal diet; ZL+S: ZL on high-salt diet; ZDF+N: ZDF on normal diet; ZDF+S: ZDF on high-salt diet.

Another important finding of our study is that SGK1 was differentially regulated in kidneys of diabetic rats as compared with normoglycemic controls. SGK1 activates and regulates the ENaC, which is the rate-limiting channel for sodium retention. ${ }^{25}$ SGK1 is known to be regulated by aldosterone and insulin. ${ }^{26}$

Under physiological conditions, a high-salt diet reduces aldosterone levels, thereby reducing SGK1 expression. Consequently, plasma sodium concentrations and BP are kept relatively stable, as seen in normoglycemic ZL rats. However, in diabetic ZDF rats, SGK1 expression was pathologically upregulated under high-salt conditions, which was paralleled by $\mathrm{ENaC} \alpha$ expression. Even at the protein level, the high-salt diet was not able to suppress SGK1 expression in ZDF rats. In addition, NCC mRNA was downregulated under high-salt conditions in ZL rats but not in ZDF rats. The fact that, in contrast to $\mathrm{ENaC}, \mathrm{NCC}$ was only downregulated in ZL rats but not upregulated in ZDF rats under high-salt conditions might be due to differences in gene regulation. Thus, NCC expression may depend on both aldosterone and renin/angiotensin II. Interestingly, a recent study showed a similar gene expression pattern in another model of salt-sensitive hypertension. SGK1 expression on a high-salt diet was downregulated 
Table 3 mRNA data of sodium transporters in the kidney

\begin{tabular}{lrclll}
\hline & \multicolumn{1}{c}{$Z L+N$} & \multicolumn{1}{c}{$Z L+S$} & \multicolumn{1}{c}{$Z D F+N$} & \multicolumn{1}{c}{$Z D F+S$} & P-value \\
\hline $\mathrm{ENaC} \alpha$ & $1.02 \pm 0.01$ & $0.80 \pm 0.04^{* *}$ & $1.02 \pm 0.27$ & $1.75 \pm 0.09^{+* \dagger}$ & 0.006 \\
$\mathrm{ENaC} \beta$ & $1.0 \pm 0.02$ & $1.04 \pm 0.11$ & $0.86 \pm 0.08$ & $0.97 \pm 0.08$ & 0.15 \\
$\mathrm{ENaC} \gamma$ & $1.02 \pm 0.02$ & $0.83 \pm 0.17$ & $0.90 \pm 0.06$ & $0.93 \pm 0.05$ & 0.56 \\
$\mathrm{NCC}$ & $1.06 \pm 0.11$ & $0.56 \pm 0.11^{* *}$ & $0.65 \pm 0.10^{* *}$ & $0.58 \pm 0.05^{* *}$ & 0.007 \\
$\mathrm{NKCC2}$ & $1.02 \pm 0.08$ & $1.16 \pm 0.14$ & $0.89 \pm 0.25$ & $0.63 \pm 0.14$ & 0.19 \\
$\mathrm{NHE3}$ & $1.02 \pm 0.02$ & $0.95 \pm 0.08$ & $0.78 \pm 0.12$ & $0.53 \pm 0.08^{\dagger}$ & 0.003
\end{tabular}

Abbreviations: $\mathrm{ENaC} \alpha$, epithelial sodium channel- $\alpha$; ZDF, Zucker diabetic fatty; ZL, Zucker lean; $\mathrm{ZL}+\mathrm{N}$, ZL on normal diet; ZL+S, ZL on high-salt diet; ZDF+N, ZDF on normal diet; ZDF+S, ZDF on high-salt diet.

Post hoc testing: ${ }^{* *} P<0.01$ vs. $\mathrm{ZL}+\mathrm{N},{ }^{\dagger} P<0.05$ vs. $\mathrm{ZDF}+\mathrm{N}$, ${ }^{\dagger \dagger} P<0.001$ vs. $\mathrm{ZDF}+\mathrm{N}$.

in Dahl salt-resistant rats but upregulated in Dahl salt-sensitive rats, a finding that was also paralleled by ENaC mRNA expression levels. ${ }^{27}$ It was previously demonstrated by the same group that ENaC and SGK1 are pathologically altered by aldosterone in Dahl salt-sensitive rats. ${ }^{28}$ Therefore, it is possible that abnormal regulation of SGK1 by aldosterone may also contribute to salt-sensitive BP in ZDF rats.

Recently, a nuclear repressor complex (Dotla) and its influence on SGK1 and ENaC were described. ${ }^{29,30}$ Besides the impact of other pathways, such as that involving Dotla, the localization of the examined sodium transporter may also account for our results. In contrast to $\mathrm{ENaC}$ and NCC, which are both part of the aldosteronesensitive distal nephron, NKCC2 is mainly located in the thick ascending limb of Henle's loop, and microdissection studies have detected NHE3 in the thick ascending limbs and the thin descending limbs, but not in the collecting duct. ${ }^{31}$

In our model, hyperinsulinemia may display another mechanism by which SGK1 is activated in ZDF rats: by phosphoinositide 3-kinase. In our experiment, SGK1 levels were not different in ZL and ZDF rats on the normal diet. Also, the insulin levels, with exhaustion of $\beta$-cell function, were only slightly elevated at this late stage of diabetes. Interestingly, in our experiment, blood glucose levels were lower in salt-loaded ZDF rats as compared with ZDF rats on the normal-salt diet. This low glucose level may be explained by elevated SGK1 expression on the high-salt diet. A study on SGK1-knockout mice and their wild-type littermates, which were not diabetic, suggested that high salt decreases SGK1 abundance and thus decreases SGK1dependent cellular glucose uptake. ${ }^{32}$ Thus, the abnormal upregulation of SGK1 in diabetic animals under high-salt conditions in our study may explain the enhanced glucose uptake into skeletal muscle and the reduced blood glucose levels under high-salt conditions. Moreover, a potential role for SGK1 in renal tubular glucose transport in diabetic kidneys was recently illustrated by Ackermann et al. ${ }^{33}$

In this study, we showed that BP in diabetic ZDF rats is salt sensitive and leads to marked vascular remodeling only on a high-salt diet. Low-renin hyperaldosteronism and altered regulation of $\mathrm{ENaC} \alpha$, a SGK1-dependent renal sodium transporter, may be important for altered sodium handling and thus for the salt sensitivity of this model.

\section{CONFLICT OF INTEREST}

The authors declare no conflict of interest.

\section{ACKNOWLEDGEMENTS}

We thank Gabriela Pietrzyk and Tatjana Libeld for excellent technical assistance. This study was supported by a grant from the Deutsche Forschungsgemeinschaft (EN 472/4-1).
1 Endemann DH, Schiffrin EL. Nitric oxide, oxidative excess, and vascular complications of diabetes mellitus. Curr Hypertens Rep 2004; 6: 85-89.

2 Pamidimukkala J, Jandhyala BS. Effects of salt rich diet in the obese Zucker rats: studies on renal function during isotonic volume expansion. Clin Exp Hypertens 2004; 26: 55-67.

3 Fujiwara K, Hayashi K, Matsuda H, Kubota E, Honda M, Ozawa Y, Saruta T. Altered pressure-natriuresis in obese Zucker rats. Hypertension 1999; 33: 1470-1475.

4 Reddy SR, Kotchen TA. Dietary sodium chloride increases blood pressure in obese Zucker rats. Hypertension 1992; 20: 389-393.

5 Chen J, Gu D, Huang J, Rao DC, Jaquish CE, Hixon JE, Chen CS, Chen J, Lu F, Hu D, Rice T, Kelly TN, Hamm LL, Whelton PK, He J, for the GenSalt Collaborative Research Group. Metabolic syndrome and salt sensitivity of blood pressure in non-diabetic people in China: a dietary intervention study. Lancet 2009; 373: 829-835.

6 Fredersdorf S, Endemann DH, Luchner A, Heitzmann D, Ulucan C, Birner C, Schmid P, Stoelcker B, Resch M, Muders F, Riegger GAJ, Weil J. Increased aldosterone levels in a model of type 2 diabetes mellitus. Exp Clin Endocrinol Diabetes 2009; 117: 15-20.

7 Bhargava A, Fullerton MJ, Myles K, Purdy TM, Funder JW, Pearce D, Cole TJ. The serum- and glucocorticoid-induced kinase is a physiological mediator of aldosterone action. Endocrinology 2001; 142: 1587-1594.

8 Muller OG, Parnova RG, Centeno G, Rossier BC, Firsov D, Horisberger JD. Mineralocorticoid effects in the kidney: correlation between alphaENaC, GILZ, and Sgk-1 mRNA expression and urinary excretion of $\mathrm{Na}+$ and $\mathrm{K}+$. J Am Soc Nephrol 2003; 14: 1107-1115.

9 Tong Q, Booth RE, Worrell RT, Stockand JD. Regulation of $\mathrm{Na}^{+}$transport by aldosterone: signaling convergence and cross talk between the PI3-K and MAPK $1 / 2$ cascades. Am J Physiol Renal Physiol 2004; 286: F1232-F1238.

10 Kobayashi T, Cohen P. Activation of serum- and glucocorticoid-regulated protein kinase by agonists that activate phosphatidylinositide 3-kinase is mediated by 3-phosphoinositide-dependent protein kinase-1 (PDK1) and PDK2. Biochem J 1999; 339: 319-328.

11 Debonneville C, Flores SY, Kamynina E, Plant PJ, Tauxe C, Thomas MA, Munster C, Chraibi A, Pratt JH, Horisberger JD, Pearce D, Loffing J, Staub O. Phosphorylation of Nedd4-2 by SGK 1 regulates epithelial $\mathrm{Na}(+)$ channel surface expression. EMBO 2001; 20: 7052-7059.

12 Snyder PM, Olson DR, Thomas BC. Serum and glucocorticoid-regulated kinase modulates Nedd4-2-mediated inhibition of the epithelial Na+ channel. J Biol Chem 2002; 277: 5-8.

13 Itani OA, Stokes JB, Thomas CP. Nedd4-2 isoforms differentially associate with ENaC and regulate its activity. Am J Physiol Renal Physiol 2005; 289: F334-F346.

14 Rozansky DJ, Cornwall T, Subramanya AR, Rogers S, Yang YF, David LL, Zhu X, Yang $\mathrm{CL}$, Ellison $\mathrm{DH}$. Aldosterone mediates activation of the thiazide-sensitive $\mathrm{Na}-\mathrm{Cl}$ cotransporter through an SGK1 and WNK4 signaling pathway. J Clin Invest 2009; 119: 2601-2612.

15 Blazer-Yost BL, Nofziger C. The role of the phosphoinositide pathway in hormonal regulation of the epithelial sodium channel. Adv Exp Med Biol 2004; 559: 359-368.

16 Wang J, Barbry P, Maiyar AC, Rozansky DJ, Bhargava A, Leong M, Firestone GL, Pearce D. SGK integrates insulin and mineralocorticoid regulation of epithelial sodium transport. Am J Physiol Renal Physiol 2001; 280: F303-F313.

17 Park J, Leong ML, Buse P, Maiyar AC, Firestone GL, Hemmings BA. Serum and glucocorticoid-inducible kinase (SGK) is a target of the PI 3-kinase-stimulated signaling pathway. EMBO J 1999; 18: 3024-3033.

18 Tiwari S, Nordquist L, Halagappa VK, Ecelbarger CA. Trafficking of ENaC subunits in response to acute insulin in mouse kidney. Am J Physiol Renal Physiol 2007; 293: F178-F185.

19 Park JB, Schiffrin EL. Small artery remodeling is the most prevalent (earliest?) form of target organ damage in mild essential hypertension. J Hypertens 2001; 19: 921-930.

20 Rizzoni D, Porteri E, Boari GE, De Ciuceis C, Sleiman I, Muiesan ML, Castellano M, Miclini M, Agabiti-Rosei E. Prognostic significance of small-artery structure in hypertension. Ciculation 2003; 108: 2230-2235.

21 Fredersdorf S, Thumann C, Ulucan C, Griese DP, Luchner A, Riegger GA, Kromer EP, Weil J. Myocardial hypertrophy and enhanced left ventricular contractility in Zucker Diabetic Fatty rats. Cardiovasc Pathol 2004; 13: 11-19.

22 Bergler T, Stoelcker B, Jeblick R, Reinhold SW, Wolf K, Riegger GA, Krämer BK. High osmolality induces the kidney-specific chlorid channel CLC-K1 by a serum and glucocorticoid-inducible kinase 1 MAPK pathway. Kidney Int 2008; 74: 1170-1177.

23 Endemann DH, Touyz RM, Iglarz M, Savoia C, Schiffrin EL. Eplerenone prevents saltinduced vascular remodeling and cardiac fibrosis in stroke-prone spontaneously hypertensive rats. Hypertension 2004; 43: 1252-1257.

24 Ehrhart-Bornstein M, Lamounier-Zepter V, Schraven A, Langenbach J, Willenberg HS, Barthel A, Hauner H, McCann SM, Scherbaum WA, Bornstein SR. Human adipocytes secrete mineralocorticoid-releasing factors. Proc Natl Acad Sci USA 2003; 100: 14211-14216.

25 Loffing J, Zecevic M, Féraille E, Kaissling B, Asher C, Rossier BC, Firestone GL, Pearce $D$, Verrey $\mathrm{F}$. Aldosterone induces rapid apical translocation of $\mathrm{ENaC}$ in early portion of renal collecting system: possible role of SGK. Am J Physiol Renal Physiol 2001; 280: F675-F682.

26 Chen SY, Bhargava A, Mastroberardino L, Meijer OC, Wang J, Buse P, Firestone GL, Verrey $F$, Pearce $D$. Epithelial sodium channel regulated by aldosterone-induced protein sgk. Proc Natl Acad Sci USA 1999; 96: 2514-2519.

27 Aoi W, Niisato N, Sawabe Y, Miyazaki H, Tokuda S, Nishio K, Yoshikawa T, Marunaka Y. Abnormal expression of ENaC and SGK 1 mRNA induced by dietary sodium in Dahl salt-sensitively hypertensive rats. Cell Biol Int 2007; 31: 1288-1291. 
28 Aoi W, Niisato N, Sawabe Y, Miyazaki H, Marunaka Y. Aldosterone-induced abnormal regulation of ENaC and SGK 1 in Dahl salt-sensitive rat. Biochem Biophys Res Commun 2006; 341: 376-381.

29 Zhang W, Xia X, Reisenauer MR, Rieg T, Lang F, Kuhl D, Vallon V, Kone BC. Aldosterone-induced Sgk1 relieves Dotla-Af9-mediated transcriptional repression of epithelial $\mathrm{Na}+$ channel alpha. J Clin Invest 2007; 117: 592-595.

30 Zhang D, Yu ZY, Cruz P, Kong Q, Li S, Kone BC. Epigenetics and the control of epithelial sodium channel expression in collecting duct. Kidney Int 2009; 75: 260-267.
31 Nishinaga H, Komatsu R, Doi M, Fustin JM, Yamada H, Okura R, Yamaguchi Y, Matsuo $\mathrm{M}$, Emoto $\mathrm{N}$, Okamura $\mathrm{H}$. Circadian expression of the $\mathrm{Na}+\mathrm{H}+$ exchanger $\mathrm{NHE3}$ in the mouse renal medulla. Biomed Res 2009; 30: 87-93.

32 Boini KM, Hennige AM, Huang DY, Friedrich B, Palmada M, Boehmer C, Grahammer F, Artunc F, Ullrich S, Avram D, Osswald H, Wulff P, Kuhl D, Vallon V, Häring HU, Lang F. Serum- and glucocorticoid-inducible kinase 1 mediates salt sensitivity of glucose tolerance. Diabetes 2006; 55: 2059-2066.

33 Ackermann TF, Boini KM, Völkl H, Bhandaru M, Bareiss PM, Just L, Vallon V, Amann K, Kuhl D, Feng Y, Hammes HP, Lang F. SGK1-sensitive renal tubular glucose reabsorption in diabetes. Am J Physiol Renal Physiol 2009; 296: F859-F866. 